\title{
The Surprising Nature of kpc-scale Quasar Jets as Revealed by Gamma-ray Observations
}

\author{
Eileen Meyer*t \\ STSCI \\ E-mail: meyerestsci.edu \\ Markos Georganopoulos \\ $U M B C$
}

William B. Sparks

STSCI

\begin{abstract}
The physical origin of the X-ray emssion from radio knots in powerful quasar jets has been a long-standing mystery. Though we know that these jets start out on the sub-pc scale as highly relativistic flows, we do not have any direct measurement of their speeds on the kpc scale, where the vast distances from the core necessitate in situ particle acceleration. If the jets remain highly relativistic, then the X-rays could be due to inverse-Compton upscattering of CMB photons. However, the IC/CMB explanation also predicts a high level of gamma-ray emission. Our recent work shows that this emission is not seen to a very high significance ( $>99.9 \%$ confidence) in at least two sources, 3C 273 and PKS 0637-752. Further, our findings suggest that these jets are not highly relativistic and that the $\mathrm{X}$-rays are synchrotron in origin. I will discuss some of the surprising implications of these findings for the energetics and radiative output of powerful quasars.
\end{abstract}

10th INTEGRAL Workshop: A Synergistic View of the High-Energy Sky

15-19 September 2014

Annapolis, MD, USA

\footnotetext{
${ }^{*}$ Speaker.

${ }^{\dagger}$ Postdoctoral Fellow, Space Telescope Science Institute | 3700 San Martin Dr Baltimore, MD 21218
} 


\section{Introduction}

In August 1999, the Chandra X-ray Observatory observed its first celestial target, quasar PKS 0637-752, during the initial focusing of the telescope (Chartas et al., 2000; Schwartz et al., 2000). Along with the bright quasar core, Chandra unexpectedly detected X-rays from the kiloparsec scale relativistic jet (previously known from radio imaging). Unlike the synchrotron spectrum of lower-power FR I jets like M87 which easily extend up to X-ray energies, the synchrotron spectrum of powerful quasar jets (including PKS 0637-752) were known to peak at or below the IR/Optical band. The X-rays detected in the kpc-scale jet of PKS 0637-752 were orders of magnitude brighter than expected from the radio-optical synchrotron spectrum, or indeed from either synchrotron self-Compton (SSC) or inverse Compton upscattering of ambient CMB photons (IC/CMB) under equipartition conditions (Chartas et al., 2000). Further, the X-ray spectrum of the jet was remarkably hard, with a photon index of $1.76 \pm 0.1$.

Proper motions measurements of sub-parsec scale AGN jets with Very Long Baseline Interferometry (VLBI) have detected superluminal proper motions which imply that these jets start out highly relativistic, with Lorentz factors $(\Gamma)$ of 10-50 (Jorstad et al., 2005; Lister et al., 2009). Though it had long been supposed based on population studies that jets decelerate and are at most mildly relativistic by the time they reach the kpc scale (e.g. Arshakian \& Longair, 2004; Mullin \& Hardcastle, 2009), no direct measurements have confirmed this generally. Tavecchio et al. (2000) and Celotti et al. (2001) thus suggested that the anomalous X-rays could be explained by IC/CMB emission if the jet remained highly relativistic $(\Gamma \sim 10)$, and was pointed at a fairly small angle to our line of sight $\left(6^{\circ}\right)$. This produces a much larger Doppler boosting factor $(\delta \sim 10)$ and enables the IC/CMB X-rays to match the observations.

Over the past decade and a half since the launch of Chandra, dozens more kpc-scale quasar jets with anomalously hard and/or high X-rays have been detected, including, notably, the nearby quasar 3C 273 (see surveys by Marshall et al. (2011, 2005); Sambruna et al. (2004) and the review by Harris \& Krawczynski (2006)). The IC/CMB model has been by far the most popular explanation of these X-rays, though problems have been noted. Besides the unconfirmed fast speeds required on the $\mathrm{kpc}$ scale, IC/CMB often requires the jet to be pointed very close along our lineof-sight, leading to a deprojected jet length longer than $1 \mathrm{Mpc}$, the upper limit for jets observed in the plane of the sky. Further, the electrons responsible for upscattering the CMB into the Chan$d r a$ band are at much lower energies than are traced by radio observations. This extension of the electron energy distribution is energetically costly, in some cases leading to 'super-Eddington' jet power requirements (Dermer \& Atoyan, 2004; Uchiyama et al., 2006). These problems lead to the suggestion that the X-rays could simply be synchrotron emission from a second electron population in the jet, albeit of unknown origin (Hardcastle, 2006; Harris et al., 2004; Jester et al., 2006; Kataoka \& Stawarz, 2005; Uchiyama et al., 2006)

Despite a significant effort by the community to amass high-resolution radio, optical, and $\mathrm{X}$-ray imaging of dozens of quasar jets, the fundamental problem up to now has been that SEDfitting alone cannot distinguish between the IC/CMB and synchrotron explanations for the X-rays. The difference in power requirements between the two mechanisms is great, as is the extremely different idea of jet structure that they imply. Discriminating between these models is essential to make progress on the actual impact of jets on their environment, and all the attendant considerations regarding galaxy and cluster evolution. 


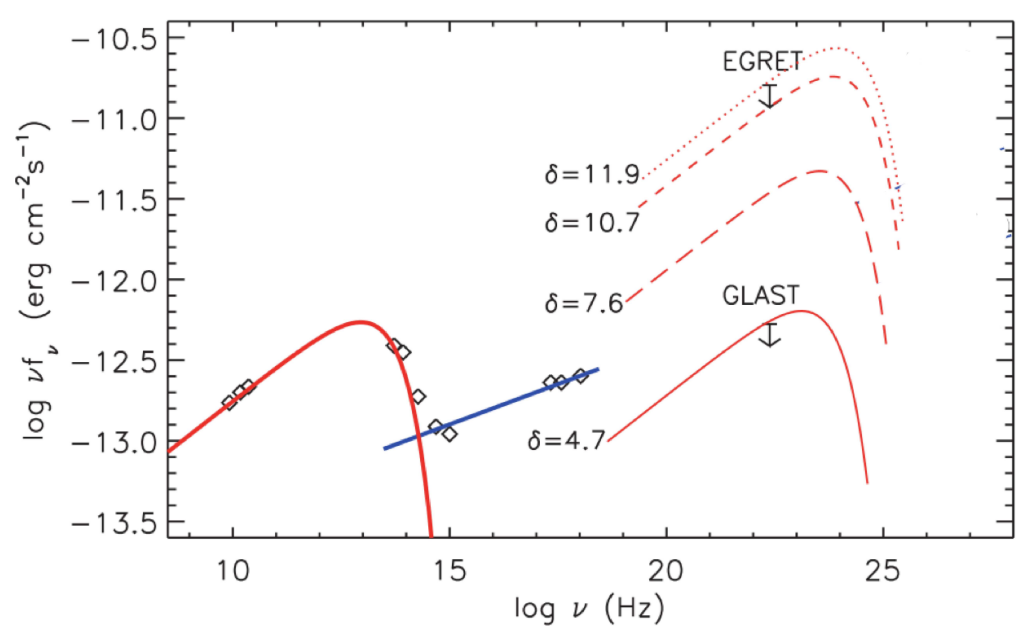

Figure 1: The radio-to-X-ray SED for the total knot emission of 3C 273 (data points shown as diamonds). The thick red curve is a fit to the radio-optical synchrotron, while the dashed/thin curves show the placement of different IC/CMB spectra depending on the Doppler boosting factor (an equipartition magnetic field is assumed to derive the values of $\delta$ ). Note that using IC/CMB to fit the X-rays fixes the ratio of $\delta / B$ and sets the level of expected gamma-ray emission without any freedom. The limit from EGRET is shown, as well as the expected sensitivity limit for the GLAST (now Fermi) mission. Adapted from Georganopoulos et al. (2006).

It was with an eye to resolving this long-standing deadlock that Georganopoulos et al. (2006) (hereafter G06) suggested that upcoming GLAST (now Fermi) observations could confirm or rule out the popularly assumed IC/CMB origin, by detecting (or not) the high level of gamma-rays implied by the IC/CMB model. This is depicted in Figure 1, where the broad-band SED for the knots of 3C 273 is shown, with a red curve showing a fit to the radio-optical synchrotron emission, and a dashed curve showing the IC/CMB model fit at different levels of beaming. As noted by G06, the shape of the IC/CMB spectrum is constrained to match the synchrotron spectrum, with a shift in frequency proportional to $(\delta / B)^{4}$ and in luminosity proportional to $(\delta / B)^{2}$, where B is the magnetic field. The simplicity of the IC/CMB model means that the value of $\delta / B$ is uniquely determined by the requirement to match the X-ray flux level, at which point there is no freedom at all in the rest of the spectrum (notably, in the Fermi band). The flux levels expected for sources like 3C 273 and PKS 0637-752 are easily reachable by Fermi over the lifetime of the mission.

Here we report Fermi observations for 3C 273 and PKS 0637-752 which show that the expected gamma-ray emission under the IC/CMB model is not detected to a high level of significance. Further, our upper limits place interesting constraints on the Doppler beaming factor which implies that these jets are not highly relativistic on the kpc scale. We also discuss the surprising implications of slow, synchrotron X-ray jets on our understanding of the total radiative output of quasars, especially at $\mathrm{TeV}$ energies.

\section{Methods}

\subsection{Fermi Analysis}

We briefly describe here the method of Fermi data analysis which was used to derive limits on the IC/CMB emission for both 3C 273 and PKS 0637-752. The analysis of 3C 273 was previ- 
ously reported in Meyer \& Georganopoulos (2014), hereafter MG14, and the general approach is the same for both sources. The specific details noted below apply to PKS 0637-752 (Meyer et al., in prep). The public scripts mentioned below are available at http://fermi.gsfc.nasa.gov/ssc/data/analysis/user/. We started by combining the all-sky weekly event files and spacecraft files for weeks 9 through 325, corresponding to Fermi Mission Elapsed Time (MET) from 239557417 to 430608212 and calendar dates 4 August 2008 to 24 August 2014.

\subsection{Localizing New Gamma-ray Sources in the ROI}

The initial step is to simply take the entire 6-year data set and analyze the region around PKS 0637-752. We use the publicly available 'quickAnalysis' script to generate the filtered event file, livetime cube, and exposure map, using a region of interest (ROI) of $10^{\circ}$ and an otherwise default configuration. The starting source list was generated from the publicly available make2FGLxml script, which generates the xml file pre-populated with 2FGL catalog sources. We use the 'quickLike' script to find an initial fit for the catalog sources in our ROI.
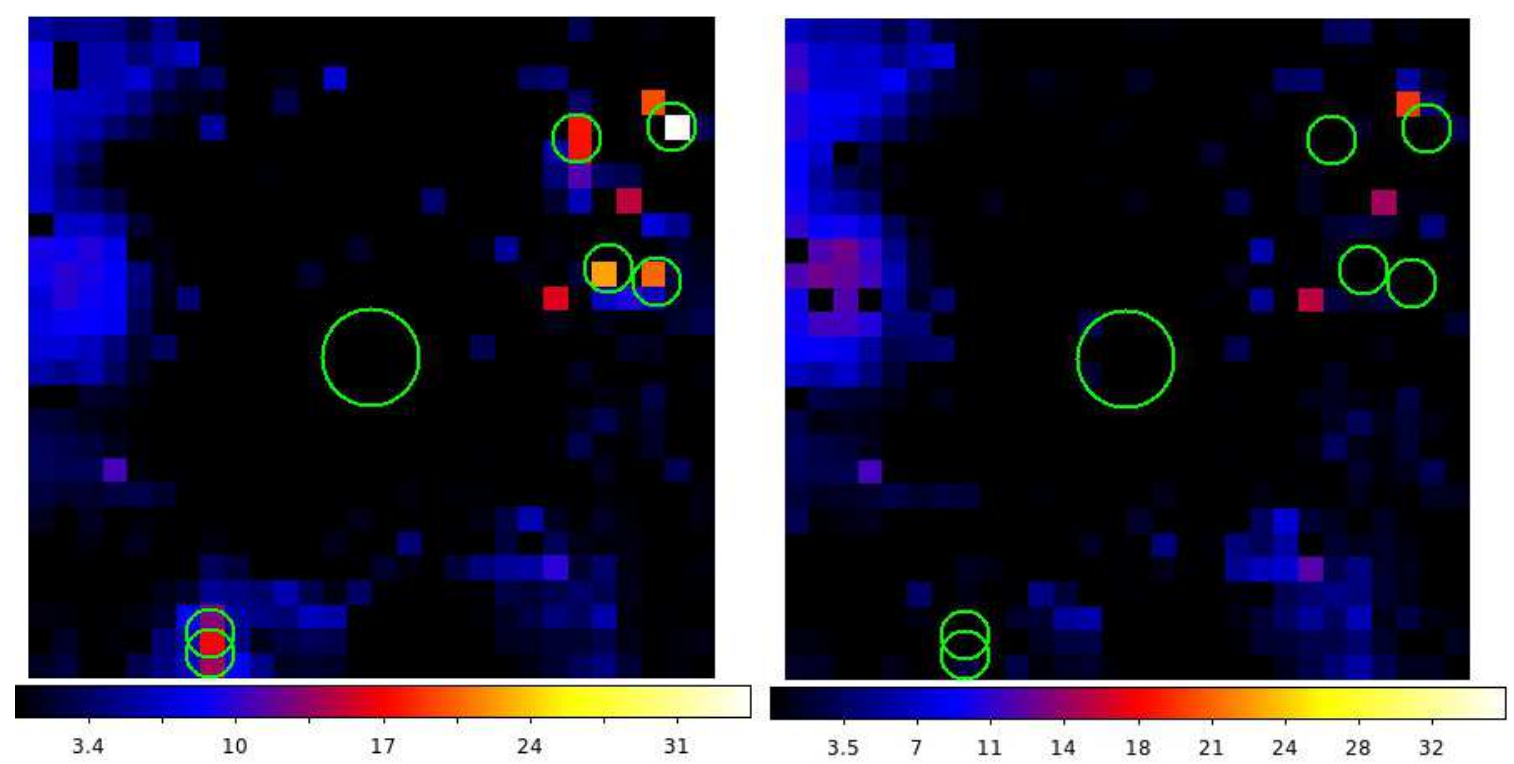

Figure 2: Left: An initial TS map of the PKS 0637 region, showing the excess TS present in $0.5^{\circ}$ pixels over the best-fit likelihood model using 2FGL catalog sources. The large circle marks the position of PKS 0637-752. The smaller circles mark the positions of the six new sources not present in the 2FGL catalog (corresponding to regions of excess TS with pixel values >20). Right: The updated TS map (same FOV and binning) after the six sources were localized and fit with a binned likelihood.

We then checked for significant sources within $7^{\circ}$ of PKS 0637-752 which are not in the 2-year LAT catalog, by making a test-statistic (TS) residual map. The TS map is created by first freezing all source parameters in the initial model their best-fit values, and then checking at different locations over the ROI, what TS value a test point source at that location would be given. The resulting initial map is shown at left in Figure 2. In two areas the coarse $0.5^{\circ}$ binning did not allow separation of what appeared to be multiple components, and so a finer $0.125^{\circ}$ binning was used over these smaller areas to determine the rough location of new sources. 
Table 1: Table of new sources in PKS 0637-752 ROI

\begin{tabular}{rcr}
\hline RA (J2000) & Dec (J2000) & \multicolumn{1}{c}{ TS } \\
\hline 82.43046 & -72.74572 & 57.5 \\
81.13040 & -69.60575 & 60.5 \\
78.98804 & -72.72570 & 28.6 \\
86.33797 & -70.34640 & 39.6 \\
119.70430 & -80.70430 & 9.9 \\
118.83300 & -80.32970 & 16.1 \\
\hline
\end{tabular}

Starting from the rough (by hand) positions of apparent new sources (TS $>20$ ), We localized these potential new sources one at a time in the following manner. We first added a power-law point source at the rough location of the excess TS, and set free the fit parameters for this source as well as those within $5^{\circ}$. We ran a binned likelihood to get a starting fit for the new source + other sources in the area. The fit parameters were then frozen, so that we could optimize the RA/Dec location of the new source. The existing tool, gtfindsrc, only works for unbinned likelihood analysis, so we built our own binned version of the tool which works in the same way. Using the frozen model, we used the python minimize function in the scipy package (L-BFGS-B method) to optimize the log-likelihood value versus the RA and Dec position, given a reasonable range of about 1 degree around the starting positions noted by hand. We then update our new model source with this optimized position, and finally un-freeze it and the local sources to get an updated model. This is then the starting model for the next new source to be added, until all new sources have been localized and a final optimized fit derived. Six new sources were added to the model, and the updated TS map run from this larger source list shows that the excess TS previously seen is now gone (right panel of Figure 2). A table of the new sources with their location and TS value is given in Table 1 .

\subsection{Light Curve and Progressive Binning Analysis}

PKS 0637-752 is a significant Fermi source, and was detected in the second Fermi catalog (2FGL), as 2FGL J0635.5-7516. Our approach to detecting and/or setting limits on the IC/CMB gamma-ray emission exploits the variability of the blazar core which cannot be spatially resolved separately from the large-scale jet due to the poor angular resolution of Fermi (generally on the order of tenths of a degree to degrees). However, during times when the blazar is quiescent, the hard, steady emission should dominate at high energies. In order to build a lightcurve of the core, the full 6+ year dataset is divided into bins of equal good time interval (GTI) time, totalling 10.5 days. We then used our starting model described above and ran a binned likelihood to fit PKS 0637752 as a power-law source, with sources more than $5^{\circ}$ away fixed. The resulting lightcurve for the core over the full time range is shown in Figure 3, with the $100 \mathrm{MeV}-100 \mathrm{GeV}$ photon flux shown on top and the corresponding TS shown below.

We next began a 'progressive binning' analysis, in which the lightcurve bins were ordered from lowest to highest TS value. Of the entire set of 75 time bins, 27 showed a TS level consistent with zero for the location of PKS 0637-752. Starting from these 27 bins combined, we progressively combine the event files for these bins plus the next lowest bin in TS, then adding the next lowest 


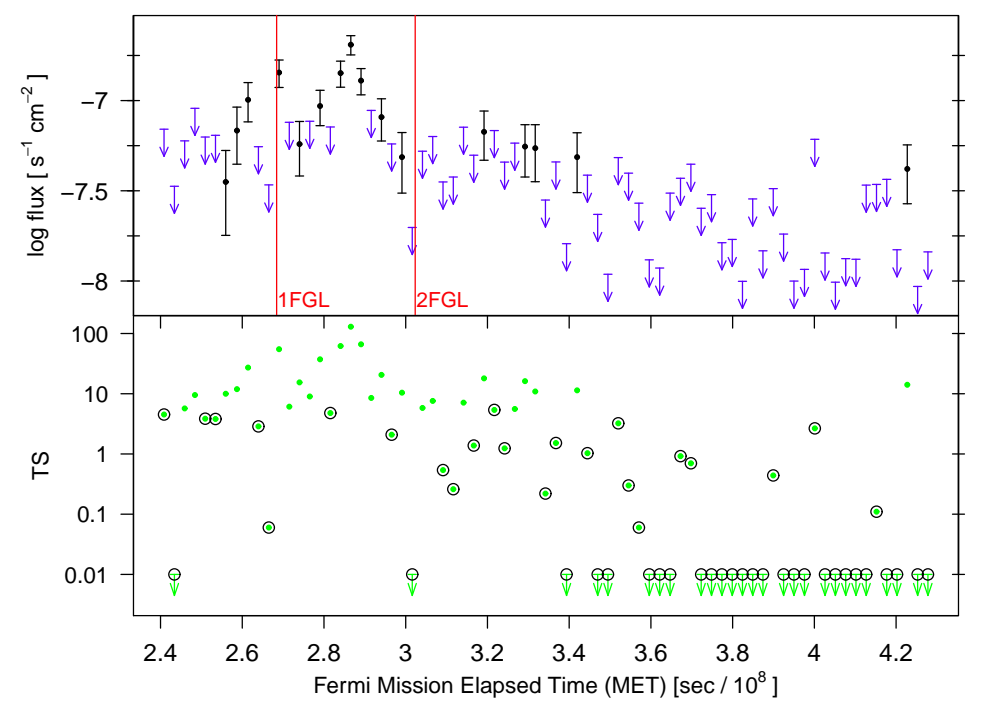

Figure 3: Upper: Lightcurve of PKS 0637-752. The total $100 \mathrm{MeV}-100 \mathrm{GeV}$ photon flux for PKS 0637-752 in 10.5-day (total GTI) bins versus the mean Mission Elapsed Time (MET) of the bin. Upper limits are shown where TS $<10$. Lower: The TS value corresponding to the same bin as above. The 50 lowest bins are circled, corresponding to the bins used to derive the lowest 3-10 GeV upper limit.

after that (etc) at each step optimizing the fit of PKS 0637-752 and the sources within $5^{\circ}$ with a binned likelihood. This re-combining of the lightcurve in a discontinuous way is appropriate for deriving a limit on the large-scale jet because the IC/CMB emission is predicted to be completely non-variable, and thus there is no risk of any selection effect via variability. The core generally dominates the flux levels determining the ordering, and is causally disconnected from the jet in any case). At each step we also evaluate the likelihood in the five canonical Fermi energy bands of $0.1-0.3,0.3-1,1-3,3-10$ and $10-100 \mathrm{GeV}$, calculating the upper limit flux value when $\mathrm{TS}<10$. Previous work on 3C 273 has shown that the exact ordering of the bins (whether by using the TS value or the total flux value for the bin) does not significantly affect the resulting upper limits.

Similar to the case of 3C 273 and in line with expectations, the highest energy bands gave upper limits which decreased with the increasing exposure as more bins are added. This is shown in Figure 4, where we have color-coded the upper limits in the 5 energy bands, and black points indicate a significant detection in the band. The decrease in upper limits going faster than $1 / \sqrt{(} t)$ is expected in the case of very low backgrounds (Meyer et al., in prep). Note that in the lowerenergy bins, where the PKS 0637-752 quasar core contributes greatly due to its soft spectrum, the upper limits reach a minimum after only a handful of bins, and after increasing for a few bins, become detections. It must be noted that the detected Fermi emission in these bands is from the quasar core, not the large-scale jet, based on the soft spectrum, and the fact that the emission level rises as more bins are added (showing that the source is indeed variable). If we were to detect the large-scale jet, we would expect it to contribute most in the two highest-energy Fermi bands, and also to remain steady in flux level after the exposure became long enough to detect it. This latter point is crucial for distinguishing a detection of IC/CMB gamma-rays versus the tail of the 


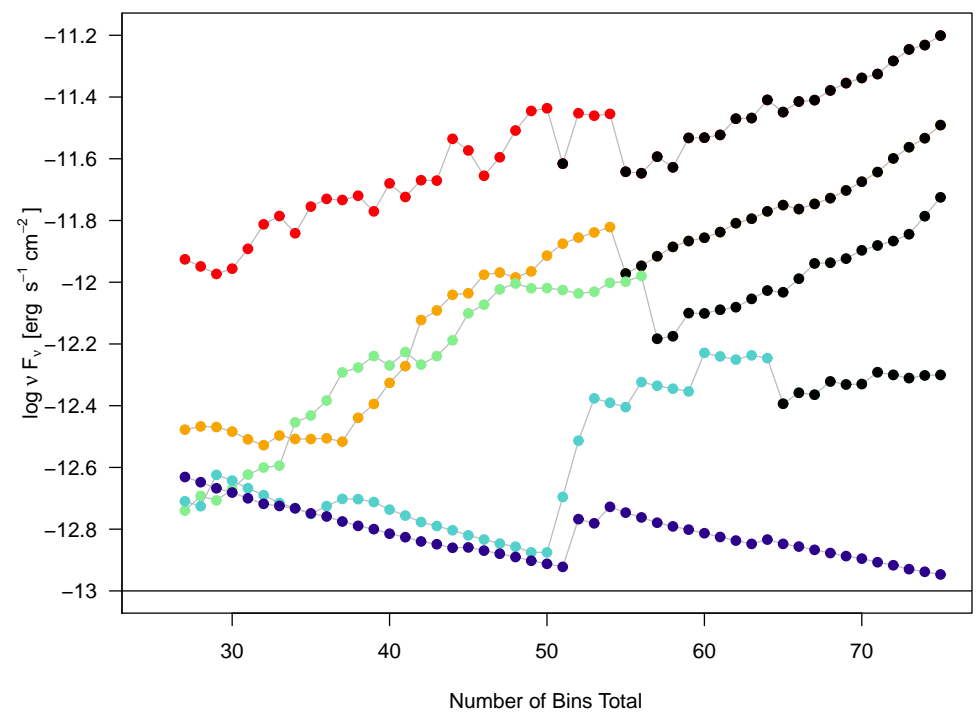

Figure 4: The upper limits/detected fluxes for each of the five Fermi energy bands (red, orange, green, cyan, navy from lowest to highest energy), where color dots correspond to upper limits and black to detections $(T S>10)$. Only for the highest bin $(10-100 \mathrm{GeV})$ is no detection ever made. The increasing fluxes indicate that the quasar core is being detected in the other bands.

Compton emission from the core, which should (like the low-energy bins) rise in flux as more bins are added. However, in the case here, the highest bin never shows a significant detection of either component. We report in Table 2 the lowest upper limit flux value reached foreach of the five bins (in $v F_{v}$, at the frequency noted).

Table 2: Lowest Upper Limits measured in Each Energy Band

\begin{tabular}{cccccc}
\hline Band & $\begin{array}{c}E_{1} \\
(\mathrm{GeV})\end{array}$ & $\begin{array}{c}E_{2} \\
(\mathrm{GeV})\end{array}$ & $\begin{array}{c}\log \text { Freq. } \\
(\mathrm{Hz})\end{array}$ & $\begin{array}{c}v F_{n} u \\
\mathrm{erg} \mathrm{s}^{-1} \mathrm{~cm}^{-2}\end{array}$ & Number of Bins Added \\
\hline 1 & 0.1 & 0.3 & 22.6 & $1.06 \mathrm{e}-12$ & 29 \\
2 & 0.3 & 1 & 23.1 & $2.96 \mathrm{e}-13$ & 32 \\
3 & 1 & 3 & 23.6 & $1.82 \mathrm{e}-13$ & 27 \\
4 & 3 & 10 & 24.1 & $1.33 \mathrm{e}-13$ & 50 \\
5 & 10 & 100 & 24.8 & $1.13 \mathrm{e}-13$ & 75 \\
\hline
\end{tabular}

\section{Results: Testing the IC/CMB Model}

We can now compare our derived limits to the expected level of gamma-ray emission from IC/CMB required in PKS 0637-752 in order to match the level of the observed X-rays. We show in Figure 5 the total radio to X-ray SED points for all of the X-ray knots combined (blue circle) as well as for the $\mathrm{X}$-ray brightest knot (wk8.9, green triangles). The solid line through blue radiooptical points represents the synchrotron spectrum used to fit the data, with a radio spectral index matching observations. As shown, by shifting this spectrum by the factors necessary to match the 
$\mathrm{X}$-rays, we over-predict the gamma-rays in four of the five Fermi energy bands. The limits shown correspond to the $95 \%$ confidence upper limits from the likelihood model. The predicted level does not depend on the assumption of equipartition magnetic fields, since the level of the X-rays sets the value of $\mathrm{B} / \delta$ which uniquely defines the entire IC spectrum when the synchrotron spectrum is known. The observed limit in the $1-3 \mathrm{GeV}$ band also violates the expected IC/CMB emission solely from X-ray-brightest knot WK8.9.
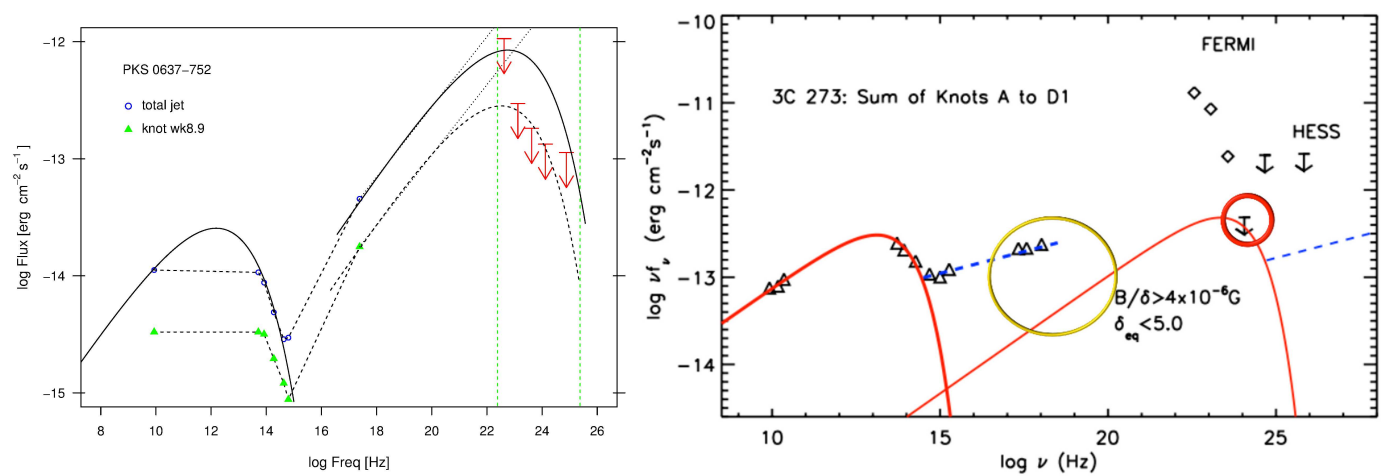

Figure 5: Left: The SED for the large-scale jet of PKS 0637-752. Data for all X-ray detected knots combined is shown as blue open circles, for X-ray brightest knot wk8.9 as green triangles. The Fermi upper limits for the gamma-ray emission violate the expected IC/CMB gamma-ray level severely for the full jet, and even for the single knot for the 1-3 GeV band. Limits shown are 95\% level. Right: The SED for the knots of 3C 273, showing again that an IC/CMB spectrum obeying the 95\% upper limit in the 3-10 GeV band cannot possibly explain the X-ray emission. Adapted from MG14.

\section{Discussion}

The result shown above for PKS 0637-752 is similar to that previously found for 3C 273 by MG14, in which the expected IC/CMB gamma-rays were ruled out at the $99.9 \%$ level in the 3-10 $\mathrm{GeV}$ energy range (right side of Figure 5). These two cases where the IC/CMB origin for the X-rays has been unambiguously ruled out join with that of PKS 1136-135, where high UV polarization has shown that the second component (UV to X-ray) must be synchrotron in origin, since significant polarization is not expected in the IC/CMB scenario (Cara et al., 2013). While this second method corroborates the general picture we believe is emerging, it is unfortunately not able to be applied in general, as few of the known quasar jets show the second component already dominating in the UV, being instead dominated by the radio-optical synchrotron component. With IC/CMB ruled out in these three cases, we are left only with the second-synchrotron origin for the anomalously high $\mathrm{X}$-ray fluxes. The existence of this second, distinct population of electrons is unexplained, but is clearly an important clue to the mechanism of particle acceleration in these jets.

\subsection{Implications for Radiative Output of kpc-scale Jets}

As the jet one-sidedness clearly indicates that the kpc-scale jets are at least mildly relativistic, it is expected that IC/CMB emission at some level cannot be avoided. Due to the very low background in the highest-energy Fermi bands, the flux limits reachable by Fermi's sky-scanning mode of operation should allow us to eventually either detect this emission or put very strong limits on the 
factor of $B / \delta$ which characterizes the flow on the kpc scale. The current limits already constrain $\delta \lesssim 5$ for both 3C 273 and PKS 0637-752, under the assumption of equipartition magnetic fields. These limits are already low enough to have interesting consequences for our understanding of the total radiative output of AGN jets on the kpc scale.

It is generally assumed that the radiative output of quasar jets is dominated by that occurring at the 'core', the base of the jet which is presumed to be very near the black hole (or $\sim$ parsecs away at most) and is therefore unresolved even in VLBI imaging. Certainly, the observed fluxes are dominated by this part of the jet due to Doppler beaming whenever the jet is pointed fairly along our line-of-sight. VLBI observations of superluminal motions place a lower limit on $\Gamma=15$ and $\Gamma=18$ for the cores of 3C 273 and PKS 0637-752, respectively (Edwards et al., 2006; Lister et al., 2013). In the left panel of Figure 6, we show the 'observed' luminosities (scaled from fluxes assuming isotropy) of both the core of 3C 273 and the summed knots of the jet, with dashed lines showing the spectral fits. The impression of the total dominance of the core is, however, mainly a product of the beaming difference between the two components. At right in Figure 6, we show on top of the same plot the beaming-corrected (i.e., true total radiated) luminosities for the core and knots as solid blue and black lines. The beaming correction is simply dividing by $\delta^{2}$ (with $\delta=15$ for the core and 5 and 1.4 for the knots). For the knots we show two curves, taking a value of $\delta=1.4$ as the minimum reasonable value based on the long-standing radio survey values to generate the highest possible level of the true knot radiated power, and apply our Fermi-derived limit of $\delta=5$ to generate the lower limit.
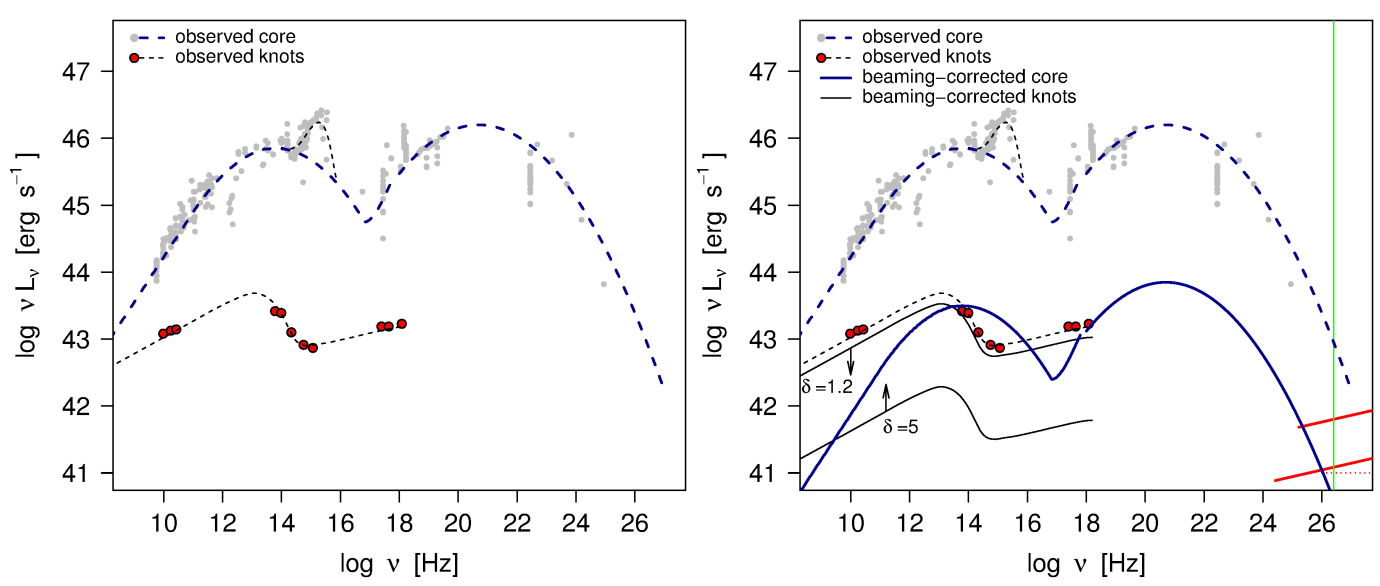

Figure 6: Left Panel: Comparing the flux and non-beaming-corrected luminosity of the 3C 273 core (gray points and blue dashed spectral fit) and total knots (red points and black dashed line). Right Panel: On the same figure as left, we have superimposed the beaming-corrected (true total luminosity) of the core (blue thick line) and knots (black thin line), where we have show the minimum and maximum beaming correction for the knots which brackets the true value. The vertical green line is drawn at an energy of $1 \mathrm{TeV}$, and we show as red solid lines the two levels of IC/CMB emission expected from the knot synchrotron X-rays for the two beaming-correction cases. In both cases, the total TeV output is greater than the typical level for TeV blazars (dashed red line).

Besides the interesting observation that the knots could dominate the radiative output (and thus contribution to unresolved backgrounds) particularly in the UV to X-rays, a further observation follows from the realization that the $\mathrm{X}$-rays are synchrotron in origin: the electrons producing the 
synchrotron $\mathrm{X}$-rays will themselves upscatter the $\mathrm{CMB}$ to produce $\mathrm{TeV}$-energy photons. These $\mathrm{TeV}$ photons will have a hard spectrum (red lines in left panel of Figure 6), and even in the minimum $\delta=1.4$ case, will produce fluxes in excess of $10^{41} \mathrm{erg} \mathrm{s}^{-1}$ which is the typical total radiative output for the canonical low-power FR I type 'TeV blazars'. However in contrast to the TeV blazars, the low beaming level does not make it easy to detect this emission, except perhaps in very nearby sources.

\section{Conclusions}

We have shown that the expected gamma-ray emission required if quasar jets produce $\mathrm{X}$-rays via IC/CMB has been ruled out in two quasar jets at a high significance level. These observations are not subject to any assumptions which might throw this result in doubt, so we are forced to fall back on a synchrotron origin for the X-rays (note however that we do not discuss here possible hadronic models, though these are energetically expensive to explain the X-rays). This considerably relaxes the power requirements for the jet away from the near and super-Eddington values, and we have shown that the limits on the IC/CMB gamma-rays constrain the jets to be relatively 'slow' on the kpc scale. This is in line with much earlier work on the size and ages of radio jets which suggested the advance speeds could not be highly relativistic on average. A very interesting outcome of the synchrotron explanation of the $\mathrm{X}$-rays is the prediction that quasar jets, and not $\mathrm{TeV}$ blazars, actually dominate the $\mathrm{TeV}$ heating in the Universe. We leave it to future work to account for how this could effect the gas heating in galaxies and clusters or possibly contribute to reionization at high redshifts. Future work also remains to see if 3C 273 and PKS 0637-752 are outliers, or if IC/CMB can be ruled out as the source of the anomalous X-rays for more quasar jets.

\section{References}

Arshakian, \& Longair. 2004, MNRAS, 351, 727

Cara et al. 2013, ApJ, 773, 186

Celotti et al. 2001, MNRAS, 321, L1

Chartas et al. 2000, ApJ, 542, 655

Dermer, \& Atoyan. 2004, ApJ, 611, L9

Edwards et al. 2006, PASJ, 58, 233

Georganopoulos et al. 2006, ApJ, 653, L5

Hardcastle. 2006, MNRAS, 366, 1465

Harris, \& Krawczynski. 2006, ARA\&A, 44, 463

Harris et al. 2004, ApJ, 615, 161

Jester et al. 2006, ApJ, 648, 900
Jorstad et al. 2005, AJ, 130, 1418

Kataoka, \& Stawarz. 2005, ApJ, 622, 797

Lister et al. 2013, AJ, 146, 120

—. 2009, AJ, 138, 1874

Marshall et al. 2011, ApJS, 193, 15

—. 2005, ApJS, 156, 13

Meyer, \& Georganopoulos. 2014, ApJ, 780, L27

Mullin, \& Hardcastle. 2009, MNRAS, 398, 1989

Sambruna et al. 2004, ApJ, 608, 698

Schwartz et al. 2000, ApJ, 540, L69

Tavecchio et al. 2000, ApJ, 544, L23

Uchiyama et al. 2006, ApJ, 648, 910 